\title{
Study on Object Detection using Open CV - Python
}

\author{
Bhumika Gupta, PhD \\ Assistant Professor, \\ C.S.E.D \\ G.B.P.E.C, Pauri \\ Uttarakhand, India
}

\author{
Ashish Chaube \\ B.Tech IV Year \\ G.B.P.E.C, Pauri \\ Uttarakhand, India
}

\author{
Ashish Negi \\ B.Tech IV Year \\ G.B.P.E.C, Pauri \\ Uttarakhand, India
}

\author{
Umang Goel \\ B.Tech IV Year \\ G.B.P.E.C, Pauri \\ Uttarakhand, India
}

\begin{abstract}
Object detection [9] is a well-known computer technology connected with computer vision and image processing that focuses on detecting objects or its instances of a certain class (such as humans, flowers, animals) in digital images and videos. There are various applications of object detection that have been well researched including face detection, character recognition, and vehicle calculator. Object detection can be used for various purposes including retrieval and surveillance. In this study, various basic concepts used in object detection while making use of OpenCV library of python 2.7, improving the efficiency and accuracy of object detection are presented.
\end{abstract}

\section{Keywords}

Object Detection, IOU, OpenCV, Python, Matlab.

\section{INTRODUCTION}

Object detection [9] and location in digital images has become one of the most important applications for industries to ease user, save time and to achieve parallelism. This is not a new technique but improvement in object detection is still required in order to achieve the targeted objective more efficiently and accurately.

The main aim of studying and researching computer vision is to simulate the behavior and manner of human eyes directly by using a computer and later on develop a system that reduces human efforts. Computer vision is such kind of research field which tries to perceive and represents the 3D information for world objects. Its main purpose is reconstructing the visual aspects of 3D objects after analyzing the $2 \mathrm{D}$ information extracted. Real life $3 \mathrm{D}$ objects are represented by $2 \mathrm{D}$ images.

The process of object detection analysis is to determine the number, location, size, position of the objects in the input image. Object detection is the basic concept for tracking and recognition of objects, which affects the efficiency and accuracy of object recognition. The common object detection method is the color-based approach, detecting objects based on their color values [4]. The method is used because of its strong adaptability and robustness, however, the detection speed needs to be improved, because it requires testing all possible windows by exhaustive search and has high computational complexity.

Object detection from a complex background is a challenging application in image processing. The goal of this project is to identify objects placed over a surface from a complex background image using various techniques. The detection of the objects can be extended using automation and robotics for plucking of the objects like apples, bananas from the corresponding tree using the image processing techniques and it will be easier, faster and convenient to pluck the apples and bananas rather than the manual plucking.

The standard performance measure that is commonly used for the object category segmentation problem is called Intersection-over-Union (IOU) [3]. Given an image, the similarities between the predicted region and the ground-truth region for an object present in the image can be found with the help of IOU measures and can be defined as the size of the intersection divided by the union of the different regions. For example, if any particular algorithm predicts each and every pixel of an image to be its background, the IOU measure can effectively penalize for that, as the intersection between the predicted and ground-truth regions would be zero, it will produce an IOU count of zero.

OpenCV library implemented in python 2.7 along with the help of Numpy is used and the world of object detection is explored, a virtual Artificial Neural Network is created using Sci-kit tool.

\section{CONCEPT}

\subsection{Theory}

Every object class has its own special features that help in classifying the object. Object recognition is that sub-domain of computer vision which helps in identifying objects in an image or video sequence. With more efficient algorithms, objects can even be recognized even when they are partially obstructed from the direct view. Various approaches to this task have been implemented in the past years.

Various terms related to object detection are:

\subsubsection{Edge matching}

- Uses edge detection techniques to find the edges

- Effect of changes in lighting and color

- Count the number of overlapping edges.

\subsubsection{Divide and Conquer search}

- All positions are to be considered as a set.

- The lower bound is determined at best position in the cell.

- The cell is pruned if the bound is too large.

- The process stops when a cell becomes small enough.

\subsubsection{Grayscale matching}

- Edges give a lot of information being robust to illumination changes. 
- Pixel distance is computed as a function of both pixel intensity and position. The same thing can compute with color too.

\subsubsection{Gradient matching}

- Comparing image gradients can also be helpful in making it robust to illumination changes.

- Matching is performed like matching greyscale images.

The ease of dealing with the image is that it is made up of pixels, so in most cases, the location of next point is easily found and can be connected with our current pixel anytime. Consider the following example for calculating Euclidean distance between center of the circle and the connected points. Take an image consisting a circle, convert it to a grayscale image, detect edges, move along edges, and draw normal which will intersect at center. Now repeat this process for entire circle or find connected edges and then calculate Euclidean distance between center of the circle and the connected points.

\subsection{Open CV}

OpenCV (Open Source Computer Vision) is an open source computer vision and machine learning software library [10]. OpenCV was initially built to provide a common infrastructure for applications related to computer vision and to increase the use of machine perception in the commercial products. As it is a BSD-licensed product so it becomes easy for businesses to utilize and modify the existing code in OpenCV.

Around 3000 algorithms are currently embedded inside OpenCV library, all these algorithms being efficiently optimized. It supports real-time vision applications. These algorithms are categorized under classic algorithms, state of art computer vision algorithms and machine learning algorithms. These algorithms are easily implemented in Java, MATLAB, Python, C, C++ etc. and are well supported by operating system like Window, Mac OS, Linux and Android.

A full-featured CUDA and OpenCL interfaces are being actively developed for the betterment of technology. There are more than 500 different algorithms and even more such functions that compose or support those algorithms. OpenCV is written natively in $\mathrm{C}++$ and has a templated interface that works seamlessly with STL containers.

For OpenCV to work efficiently with python 2.7 we need to install NumPy package first.

\subsection{NumPy}

NumPy is the fundamental package for scientific computing with Python [11].It can be treated as an extension of the Python programming language with support for multidimensional matrices and arrays. It is open source software with many contributors. It contains among other things:

- A powerful N-dimensional array object.

- Broadcasting functions.

- Tools for integrating $\mathrm{C} / \mathrm{C}++$ and FORTRAN code.

- Useful linear algebra, Fourier transform, and random number capabilities.

Besides its obvious scientific uses, NumPy can also be used as an efficient multi-dimensional container of generic data.
Arbitrary data types can be defined. This allows NumPy to seamlessly and speedily integrate with a wide variety of databases.

NumPy is licensed under the BSD license, enabling reuse with few restrictions.

\subsection{Object Classification In Moving Object Detection}

Object classification approach is based on shape, motion, color and texture. The classification can be done under various classes such as trees, animals, humans, objects etc. Tracking objects and analyzing their features is a key concept of object classification.

\subsubsection{Shape Based}

A mixture of image-based and scene-based object parameters such as image blob (binary large object) area, the aspect ratio of blob bounding box and camera zoom is given as input to this detection system. Classification is performed on the basis of the blob at each and every frame. The results are kept in the histogram.

\subsubsection{Motion Based}

When a simple image is given as an input with no objects in motion, this classification is not needed. In general, non-rigid articulated human motion shows a periodic property, hence this has been used as a strong clue for classification of moving objects. Based on this useful clue, human motion can be distinguished from other objects motion.

\subsubsection{Color Based}

Though color is not an appropriate measure alone for detecting and tracking objects, but the low computational cost of the color based algorithms makes the color a very good feature to be exploited. For example, the color-histogrambased technique is used for detection of vehicles in real-time. Color histogram describes the color distribution in a given region, which is robust against partial occlusions.

\subsubsection{Texture-Based}

The texture-based approaches with the help of texture pattern recognition work similar to motion-based approaches. It provides better accuracy, by using overlapping local contrast normalization but may require more time, which can be improved using some fast techniques.

\section{STEPS INVOLVED IN OBJECT DETECTION IN PYTHON 2.7}

Let us start with an image (im.jpg) and detect various objects in it.

\subsection{Install OpenCV-Python}

Below Python packages are to be downloaded and installed to their default location - Python-2.7.x, NumPy and Matplotlib. Install all packages into their default locations. Python will be installed to C/Python27/. Open Python IDLE. Enter import NumPy and make sure NumPy is working fine. Download OpenCV from Sourceforge. Go to OpenCV/build/python/2.7 folder. Copy cv2.pyd to C:/Python27/lib/site-packages. 


\subsection{Read an Image}

Use the function CV2.imread() to read an image. The image should be in the current working directory otherwise, we need to specify the full path of the image as the first argument. The second argument is a flag which specifies the way image should be read.

1. CV2.IMREAD_COLOR: This function is used to load a color image. Transparency of image, if present will be neglected. It is the default flag.

2. CV2.IMREAD_GRAYSCALE: Loads image in grayscale mode

3. CV2.IMREAD_UNCHANGED: Loads image as such including alpha channel.

\subsection{Feature detection and description}

- Understanding features (What are the main features in an image? How can finding those features be useful to us?)

- Corner detection (Okay, Corners are good features? But how do we find them)

- Feature matching (We know a great deal about feature detectors and descriptors. So let us now learn to match different descriptors. OpenCV provides two techniques, Brute-Force matcher, and FLANN based matcher.)

- Homography (As we are aware of feature matching, so let us now blend it with Camera calibration and $3 \mathrm{D}$ reconstruction (calib3d module) to find objects for description in a complex image.)

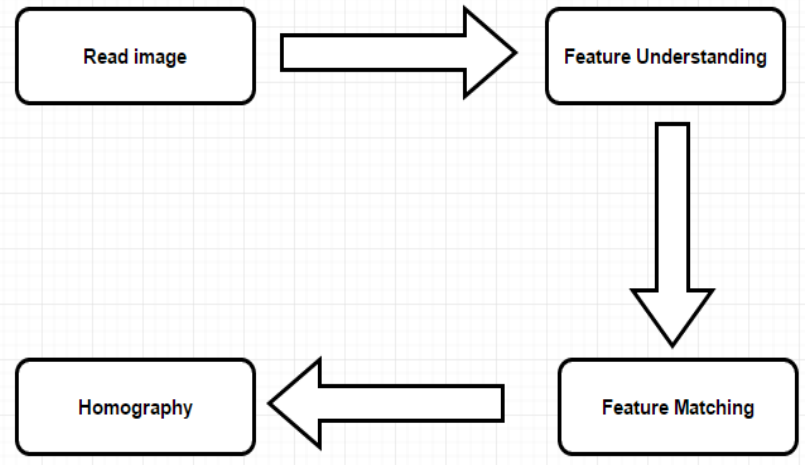

Fig: 1 Flow Chart for Object Detection

\section{PYTHON VS OTHER LANGUAGES FOR OBJECT DETECTION}

Object detection is a domain-specific variation of the machine learning prediction problem.

Intel's OpenCV library that is implemented in $\mathrm{C} / \mathrm{C}++$ has its interfaces available in a number of programming environment such as C\#, Matlab, Octave, R, Python etc.

Some of the benefits of using Python codes over other language codes for object detection are

- More compact and readable code.

- Python uses zero-based indexing.

- Dictionary (hashes) support is offered.

- Simple and elegant Object-oriented programming.
- $\quad$ Free and open.

- Multiple functions can be packaged in one module.

- More choices in graphics packages and toolsets.

\section{VARIOUS OBJECT DETECTION ALGORITHMS IMPLEMENTED IN PYTHON}

\subsection{Haar-like features}

It is an effective object detection technique which is proposed by Paul Viola and Michael Jones in 2001. It is a machine learning based method for object detection where we train a classifier from a lot of images. This classifier is then used in detecting objects in an image.

Initially, the algorithm needs images with faces (positive images) and images without faces (negative images) to train a classifier and then extract features from this classifier.

This method introduces a concept cascade of the classifier. Instead of applying all the features at once we group the features into different stages of the classifier and apply one by one. Discard the window if it fails in the first stage. If it passes the stage then continue the process. The window which passes through all the stages will be our desired region.

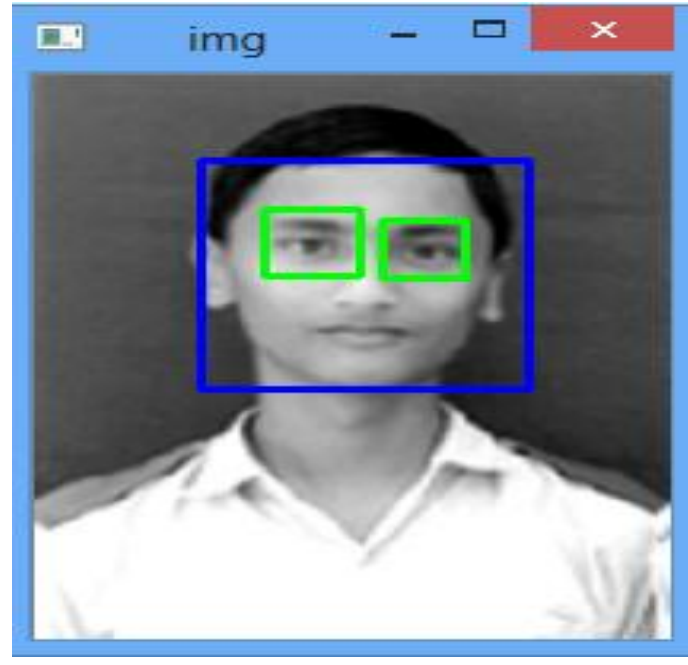

Fig:2 Face Detection using Haar Cascade

\subsection{Circular Hough Transformation}

Hough transformation was invented by Richard Duda and Peter Hart in 1992, this transformation was initially meant to detect arbitrary shapes from an image. It was later modified to detect circular objects in low-contrast noisy images and referred as Circular Hough Transformation [6].

CHT relies on equations [6] for circles:

$$
r^{2}=(x-a)^{2}+(x-b)^{2}
$$

where $\mathrm{a}$ and $\mathrm{b}$ are the coordinate of the center, and $\mathrm{r}$ is the radius of the circle.

CHT relies on three parameters, which require larger computation time and memory and it increases the complexity to extract information from the image.

For simplicity, CHT programs are provided with a constant value of radius or provided with a range of radius prior to running the application. 


\subsection{Template matching}

Template matching [1,2] \& [5] is a high-level machine vision technique to detect objects from an image that matches a given image pattern. This technique matches the source image with the template image or patch.

If the template image has strong features, the feature-based approach may be used otherwise template based approach is used.

\subsection{Blob detection}

This method is used to detect regions in an image that differs in properties. A blob is a region in the image in which all the points can be considered to be similar to each other. There are two classes of blob detection method: differential method and local extrema method.

\subsection{The Gradient-based method}

The gradient-based method uses spatial and temporal partial derivatives to estimate image flow at every position in the image. If the motion is not known in advance to be restricted to a small range of possible values then a multi- scale analysis must be applied so that the scale of the smoothing prior to derivatives estimation is appropriate to the scale of the motion. This can make this method computationally expensive.

\subsection{Local Binary Pattern}

LBP is a visual descriptor proposed in 1990. It is a powerful method for texture classification.

The LBP feature vector is created in the following manner

Divide window into cells.

- For each pixel in the cell, compare it with its 8 adjacent pixels.

- If the value of the pixel is greater than its neighbor, write " 0 ", otherwise " 1 ". This gives an 8 digit binary number which is converted to decimal for convenience

- Aggregate histogram of all the cells is calculated and compared.

- Normalize the histogram. Concatenate histogram of all cells. This gives the feature vector of the entire window.

This vector now can be used to classify the images

\subsection{Bag-of-words method}

This method can be applied to image classification. Images are treated like visual words. These visual words are important points in the images. These points are called features. This method can be used for image classification by creating a large vocabulary of many visual words and representing each histogram of the frequency words that are in the image.

\subsection{Deep Face method}

Facebook AI research group has developed Deep Face Software in Menlo Park, California by the support of an advanced deep learning neural network. A piece of software that simulates an approximation of how real neurons works is called a Neural Network. Deep Face Learning can be performed by Machine Learning. It can be defined as a huge body of data that develops a high-level abstraction by looking for recurring faces.

\section{APPLICATIONS AND FUTURE SCOPE}

Computer vision is still a developing discipline, it has not been matured to that level where it can be applied directly to real life problems.

After few years' computer vision and particularly the object detection would not be any more futuristic and will be ubiquitous. For now, we can consider object detection as a sub-branch of machine learning.

Some common and widely used application of object detection are:

\subsection{Face Detection}

Have you ever wondered how Facebook detects your face when you upload a photo? Not only it detects, it remembers the face too. This is a simple application of object detection that we see in our daily life.

\subsection{Counting objects/peoples}

Object detection can be also used for counting purpose, it is used for keeping a count of particular or all objects in an image or a frame. For e.g. from a group photograph it can count the number of persons and if implemented smartly you may also find out different people with different dresses.

\subsection{Vehicle detection}

Similarly, when the object is a vehicle, object detection along with tracking can be used for finding the type of vehicle, this application may be extended to even make a traffic calculator.

\subsection{Industries}

Object detection is also used in industrial processes for the identification of different products. Say you want your machine to only detect objects of a particular shape, you can achieve it very easily. For e.g. Hough circle detection transform [6] can be used for detecting circular objects.

\subsection{Security}

Identification of unwanted or suspicious objects in any particular area or more specifically object detection techniques are used for detecting bombs/explosives. It is also even used for personal security purpose.

\subsection{Biometric recognition}

Biometric recognition uses physical or behavioral traits of humans to recognize any individuals for security and authentication purpose [1]. It uses distinct biological traits like fingerprints, hand geometry, retina and iris patterns etc.

\subsection{Surveillance}

Objects can be recognized and tracked in videos for security purpose. Object recognition is required so that the suspected person or vehicle can be tracked [1].

\subsection{Medical analysis}

Object detection is used to detect diseases like a tumor, stones, cancer in MRI images [1].

\subsection{Optical character recognition}

Characters in scanned documents can be recognized using object recognition [1].

\subsection{Human computer interaction}

Human gestures can be stored in the system and can be used for recognition in a dynamic environment by computers to interact with humans [1]. 
Object detection's scope is not yet limited here. You can use it for any purpose you can think of. For e.g. for solving number puzzles by just giving their images as input and applying some proper algorithms after detecting different numbers and their places from the input image.

\section{CHALLENGES}

The main purpose is to recognize a specific object in real time from a large number of objects. Most recognition systems [2] are poorly scalable with many recognizable objects. Computational cost rises as the number of objects increases. Comparing and querying images using color, texture, and shape are not enough because two objects might have same attributes. Designing a recognition system with abilities to work in the dynamic environment and behave like a human is difficult. Some main challenges to design object recognition system are lighting, dynamic background, the presence of shadow, the motion of the camera, the speed of the moving objects, and intermittent object motion weather conditions etc.

\section{CONCLUSION}

The possibilities of using computer vision to solve real world problems are immense. The basics of object detection along with various ways of achieving it and its scope has been discussed. Python has been preferred over MATLAB for integrating with OpenCV because when a Matlab program is run on a computer, it gets busy trying to interpret all that Matlab code as Matlab code is built on Java. OpenCV is basically a library of functions written in $\mathrm{ClC}++$. Additionally, OpenCV is easier to use for someone with little programming background. So, it is better to start researching on any concept of object detection using OpenCV-Python.

Feature understanding and matching are the major steps in object detection and should be performed well and with high accuracy. Deep Face is the most effective face detection method that is preferred over Haar-Cascade by most of the social applications like facebook, snap chat, Instagram etc.

In the coming days, OpenCV will be immensely popular among the coders and will also be the prime requirement of IT companies. To improve the performance of object detection IOU measures are used.

\section{REFERENCES}

[1] Khushboo Khurana and Reetu Awasthi,"Techniques for Object Recognition in Images and Multi-Object Detection",(IJARCET), ISSN:2278-1323,4 ${ }^{\text {th }}$, April 2013.

[2] Latharani T.R., M.Z. Kurian, Chidananda Murthy M.V,"Various Object Recognition Techniques for Computer Vision", Journal of Analysis and Computation, ISSN: 0973-2861.

[3] Md Atiqur Rahman and Yang Wang, "Optimizing Intersection-Over-Union in Deep Neural Networks for Image Segmentation," in Object detection, Department of Computer Science, University of Manitoba, Canada, 2015.

[4] Nidhi, "Image Processing and Object Detection", Dept. of Computer Applications, NIT, Kurukshetra, Haryana, 1(9): 396-399, 2015.

[5] R. Hussin, M. Rizon Juhari, Ng Wei Kang, R.C.Ismail, A.Kamarudin, "Digital Image Processing Techniques for Object Detection from Complex Background Image,"Perlis, Malaysia: School of Microelectronic Engineering, University Malaysia Perlis, 2012.

[6] S.Bindu, S.Prudhvi, G.Hemalatha, Mr.N.Raja Sekhar, Mr. V.Nanchariah,"Object Detection from Complex Background Image using Circular Hough Transform", IJERA, ISSN: 2248-9622, Vol. 4, Issue 4(Version 1), April 2014, pp.23-28.

[7] Shaikh, S.H; Saeed, K, and Chaki.N,"Moving Object Detection Using Background Subtraction" Springer, ISBN:978-3-319-07385-9.

[8] Shijian Tang and Ye Yuan,"Object Detection based on Conventional Neural Network".

[9] (2017, January 17). Object Detection [Online].Available: http://en.m.wikipedia.org/wiki/Object_detection

[10] Opencv.org, 'About OpenCV', 2017. [Online]. Available:http://www.opencv.org/about

[11]Numpy.org, 2017. [Online]. Available: http://www.numpy.org 